\title{
Metastatic melanoma to the small bowel complicated by fistula formation
}

\author{
Nausheen Khan, MB BS, FC Rad \\ Irma van de Werke, MB ChB, FRCR \\ Department of Radiology, Kalafong Hospital, Pretoria
}

\section{Zaeem I Ebrahim, MB BCh, FC Rad Farzanah Ismail, MB BCh, FC Rad \\ Department of Radiology, Steve Biko Academic Hospital and University of Pretoria}

Corresponding author: Z Ebrahim (zaeem@iafrica.com)

\section{Introduction}

Malignant melanoma ( $\mathrm{MM})$ is an unpredictable tumour that can metastasise to any organ, and is well known for its widespread dissemination. The incidence of metastases to the gastro-intestinal (GI) tract is well documented; this, however, is a late manifestation of the disease with an overall poor prognosis. Most GI metastases are asymptomatic and are only discovered on postmortem, with the majority in the small bowel. The presenting symptoms are usually of obstruction or intussusception; GI bleeding is also common. Fistula formation with the small bowel is rare; ours is believed to be the second case documented. ${ }^{1,2}$

\section{Case history}

A 59-year-old man presented with a 2-month history of change in bowel habits. There was no significant history of illness in the past and no known primary lesion. Clinically, his abdomen was distended with a palpable right iliac fossa mass extending to the supra-pubic area. There were no signs of guarding or tenderness. Blood tests showed that he was anaemic, with haemoglobin of $9.4 \mathrm{~g} / \mathrm{dl}$, leucocytosis and raised C-reactive protein (CRP).

\section{Radiological findings}

\section{Ultrasound}

Ultrasound showed a para-caecal mass associated with para-aortic and para-pancreatic lymphadenopathy. Computed tomography (CT) scans (Figs 1 - 3) revealed a large intraperitoneal, predominantly mesenteric, mass in the right lower abdomen crossing to the contra-lateral side, with a large central ulceration and fistula formation with the distal small bowel i.e. ileum with an air contrast level within the tumour. There was also para-aortic and peri-pancreatic lymphadenopathy.The rest of the small bowel was displaced anteriorly, and the sigmoid pushed posterolaterally.

\section{Discussion}

$\mathrm{MM}$ is one of the most common malignancies to metastasise to the GI tract. ${ }^{2}$ It does so via lymphatic channels to parenchymal organs. Symptomatic metastasis to the GI tract is seen only in $2 \%$ of patients. ${ }^{3}$ Metastases may present at the time of primary diagnosis or decades later as the first sign of recurrence. MM that involves the GI tract may be either primary or metastatic. ${ }^{2}$ Symptoms include abdominal
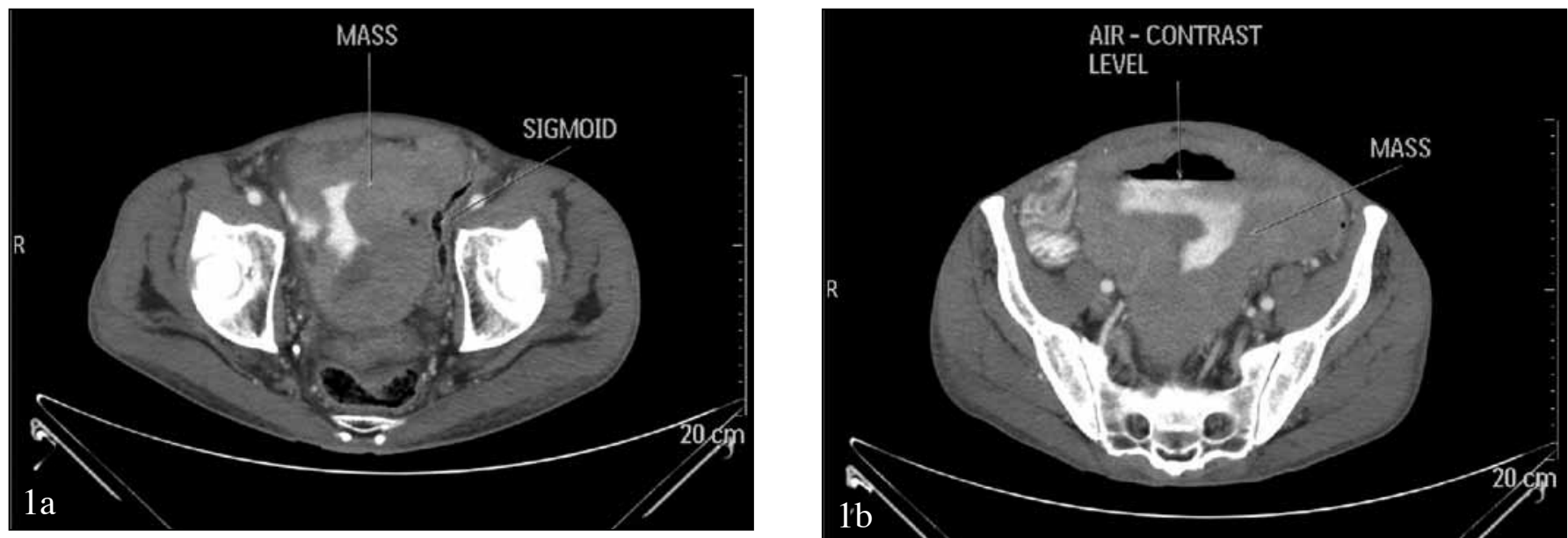

Fig. 1. Axial post-contrast CT, venous phase at the level of the pelvis, showing a large mass with central ulceration as well as contrast collection (1a) and an air fluid level (1b). 


\section{CASE REPORT}

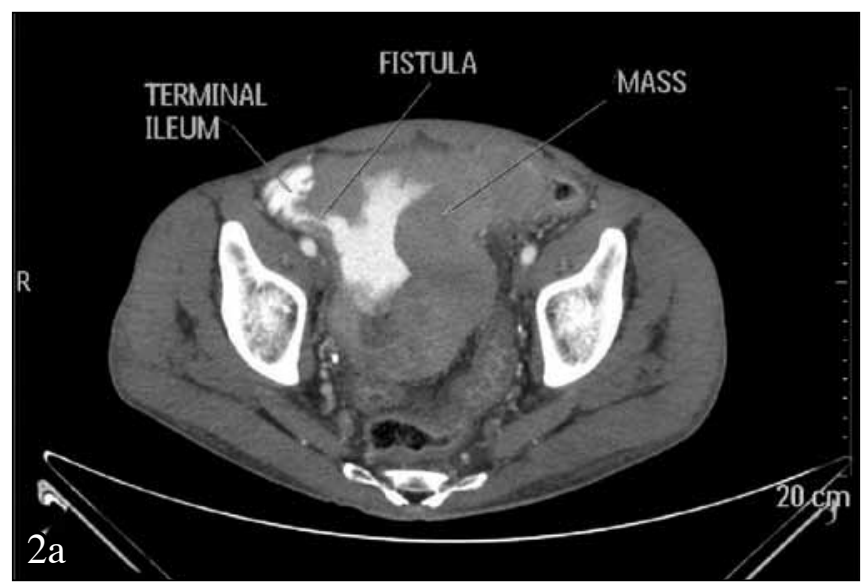

Fig. 2. Axial (2a) and coronal (2b) post-contrast CT, venous phase, showing communication between contrast-containing mass and terminal ileum.

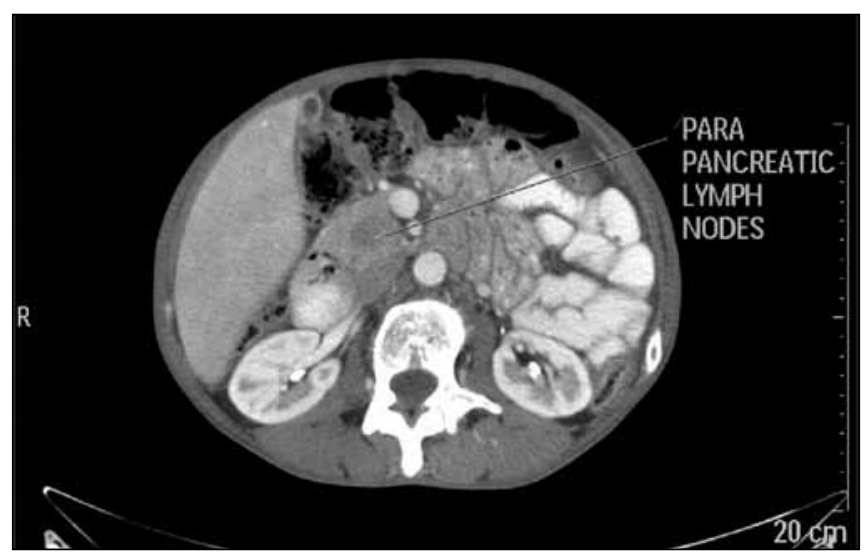

Fig. 3. Axial post-contrast CT scan, venous phase at the level of the kidneys, showing large para-pancreatic lymph nodes with central low density.

pain, dysphagia, small bowel obstruction, haematemesis and melena. Symptoms are usually identical to those caused by primary GI tumours. ${ }^{4,6}$

Primary GI melanoma can arise in various GI mucosal sites, including the oral cavity, oesophagus, small bowel, rectum and anus, in the absence of prior cutaneous melanoma. Primary melanoma of the GI tract is rarely diagnosed at an early stage. Distinguishing between a primary GI mucosal melanoma and melanoma metastases to the GI tract from an unknown or regressed cutaneous primary may be difficult. ${ }^{2,4}$

The most common sites of metastases are lymph nodes $(73 \%)$ and lung (71\%), followed by the liver, brain, bone and adrenal glands; GI metastasis stands at $43 \%$. Superficial spreading melanoma is the most common subtype to metastasise to the GI tract. $^{2}$

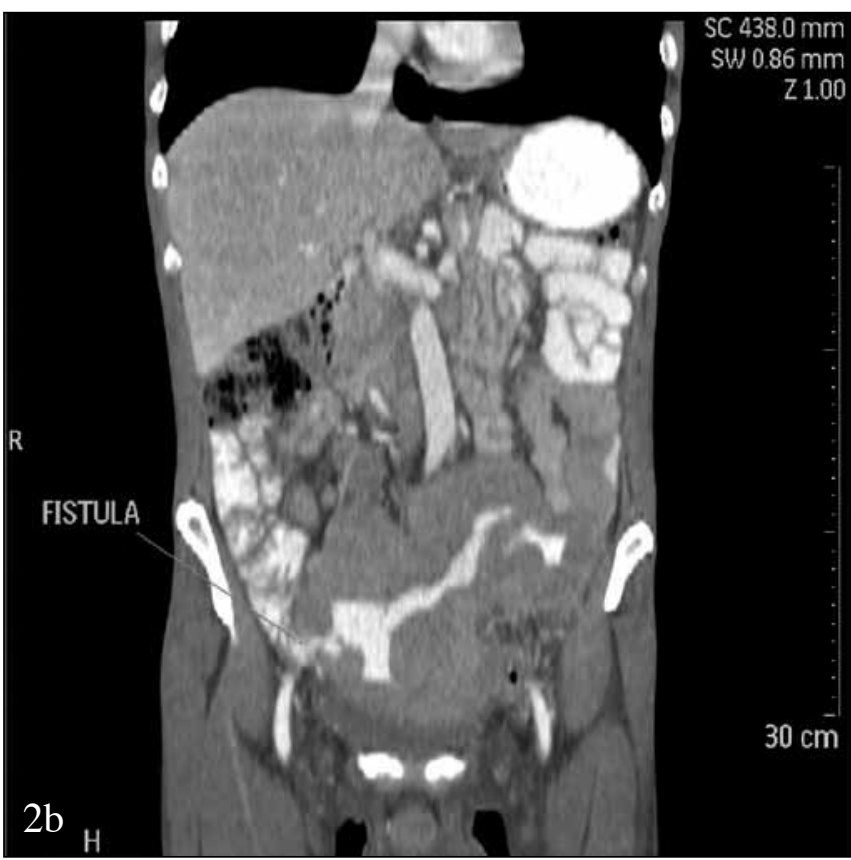

Diagnosis of MM is generally made by radiographic contrast studies, including CT, ultrasound and barium studies, as well as endoscopic evaluation. Most recently, positron emission tomography - computed tomography (PET/CT) has been used to identify sites of metastatic melanoma. CT sensitivity is between 60 and $70 \%{ }^{2,5}$

Metastatic lesions may be intraluminal masses, ulcerating lesions (as in our case), diffusely infiltrating lesions or mesenteric implants.

\section{Conclusion}

MM in the GI tract is a late manifestation of the disease, with overall poor prognosis. ${ }^{6}$ In a patient presenting with a GI tract mass, initial differentials should include primary GI tract tumours as well as lymphoma; however, a high index of suspicion for metastatic melanoma should be maintained if the patient presents with seemingly unrelated symptoms or history of treated melanoma in the past.

1. Casanova F, Lizazo J, Shezi S, Oliver F. Right inguinal bowel fistula on the course of melanoma disease. Internet Journal of Oncology. 2009;6(2).

2. Liang KV, Sanderson SO, Nowakowski GS, Arora AS. Metastatic malignant melanoma of the gastrointestinal tract. Mayo Clinic College of Medicine. Mayo Clinic Proceedings 2006;81(4): 511-516.

3. Tsilimparis N, Menenakos C, Rogalla P, Braumann C, Hartmann J. Malignant melanoma metastasis as a cause of small bowel perforation. Onkologie 2009;32(6):356-358.

4. Capizzi PJ, Donohue JH. Metastatic melanoma of the gastrointestinal tract: a review of the literature. Compr Ther 1994:20:20-23.

5. Serin G, Doğanavşargil B, Calişkan C, Akalin T, Sezak M, Tunçyürek M. Colonic malignant melanoma, primary or metastatic? Case report. Turk J Gastroenterol 2010;21(1):45-49.

6. Marks JA, Rao AS, Loren D, Witkiewicz A, Mastrangelo MJ, Berger AC. Malignant melanoma presenting as obstructive jaundice secondary to metastasis to the ampulla of Vater. J Pancreas Online 2010;11(2):173-175. 